\title{
Experimental investigation of energy dissipation mechanisms in laminated safety glass for combined blast-temperature loading scenarios
}

\author{
Tim Bermbach • Martien Teich • \\ Norbert Gebbeken
}

Received: 30 November 2015 / Accepted: 27 April 2016 / Published online: 18 May 2016

(C) Springer International Publishing Switzerland 2016

\begin{abstract}
Protective glazing is an important field of research and development as most common injuries resulting from an explosion event in urban territory are caused by the occurring shock wave and accelerated fragments of failed glass structures. In this context a selection of laminated glass specimens differing in lamination technique, extent of tempering and interlayer material is tested in shock tube experiments at the facilities of the Bundeswehr Technical Center 52 (WTD 52) in Oberjettenberg. Unlike conservative testing the specimen is subjected to a combination of blast and temperature loading. The influence of the interlayer temperature on the laminate behavior is examined according to the elastic response of the composite. Furthermore the paper focusses on the residual load carrying capacity after fracture of the glass component. An assessment of the specimen performance is undertaken by a work balance approach, which leads to the determination of energy dissipation capacities in elastic and damage configurations. This method is suitable for a further breakdown of internal work and dissipation processes. Fracture patterns are evaluated to identify crack densities along paths of principal normal stress.
\end{abstract}

T. Bermbach $(\bowtie) \cdot$ N. Gebbeken

University of the Bundeswehr Munich,

Werner-Heisenberg-Weg 39, 85577 Neubiberg, Germany

e-mail: tim.bermbach@unibw.de

M. Teich

seele GmbH, Gersthofen, Germany
The average strain and strain rates close to originated crack centers are determined. Finally the testing results are superimposed to estimate the impact of material and loading parameters on the overall performance.

Keywords Laminated glass · Blast-temperature · Energy dissipation

\section{Introduction}

The events at Paris in November 2015 and at Oslo in July 2011 show the fatal consequences of terrorism, whether motivated by Islamic fundamental or other ideological reasons, in the vulnerable environment of city centers. Within the last decade, numerous attacks could be foiled in Germany, so that they fortunately remained attempts thus far. Regarding the growing risk of terrorist acts in urban surroundings, the attention has been drawn to extraordinary loading scenarios in order to ensure the reliability of structures.

Apart from the architectural aspect, a glass structure should be designed to resist these loadings and act as passive protection. In explosion events, the hazard of failing glass elements is one of the main causes for physical injuries (Bjerketvedt and Mjaavatten 2005; Norville et al. 1999; Brismar and Bergenwald 1982). The total failure of glass structures should not lead to cuttings by accelerated fragments on the protected side. Furthermore, the failure of façade elements results in a loss of protection against the air blast wave, thermal 
loading and hot combustion products inside the buildings, which cause ear drum and lung ruptures, burns and intoxication. In a thinkable scenario, the mechanical blast loading may be superimposed by thermal loading resulting from

- the climatic conditions (ambient temperature, solar radiation),

- precedent fires,

- thermal radiation of a fireball or

- abrupt rise of the temperature due to an entropy jump across the shock wave (Salas and Iollo 1996).

An example of the comprehensive approach towards a combined blast-temperature loading can be found in Mueller et al. (2015). Martinsen and Marx (1999) present a dynamic model for the prediction of radiant heat from fireballs.

The dissipation of energy becomes a crucial factor during the response of a structure to a blast load. New design solutions have been developed to achieve adaptive-flexible supporting structures, e. g. by Wellershoff et al. (2012). However, a pane of laminated safety glass itself may have a high potential of energy dissipation within the fracture process.

Polymers are widely applied as interlayer material in glass composites. The material properties of used polymers may show to some extent a strong dependency on temperature and strain rate in the relevant range of considered exposure, which has been investigated in various publications (Juang et al. 2001; Boyce et al. 2000; Sobek et al. 2000). The lamination process and the chemical composition of the interlayer govern the strength of the bond between the components of laminated safety glass. Consequently, a variation of interlayer properties and bond strength is assumed to impact the structural behavior of the whole composite.

The focus of this paper lies on the residual load carrying capacity of laminated safety glass exposed to a combination of both blast and temperature loading. Furthermore, the aim of the research is to study the performance of laminated glass for different interlayer materials and lamination techniques. In detail, the mechanisms, which dissipate energy before total failure of the laminate, are investigated and discussed.

\section{Structural response of laminated safety glass}

The failure of laminated safety glass as a composite structure under both static and high dynamic loading occurs progressively. The structural response can be investigated and described in the intact state of the laminate and in damage configurations. This approach is suitable for the experimental evaluation. Furthermore the observed response of the structure as a whole poses the basis for smeared numerical models as shown in Larcher et al. (2012) and Hooper et al. (2012).

\subsection{Elastic configuration of the laminate}

The structural response of uncracked laminated safety glass loaded perpendicular to its plane is generally governed by the bending mode. The behavior of the undamaged laminate has been analyzed in various publications (Norville et al. 1998; Behr et al. 1993; Vallabhan et al. 1993; Hooper 1973). Wei et al. (2006) studied the elastic stress characteristics of a composite during a dynamic response. The pre-fracture response of glass subjected to blast loads is investigated by Cormie et al. (2015).

The behavior of the laminate is depending on the interlayer properties and on the applied load. Two cases limit the range of response. If the interlayer is assumed to be ideally rigid, the composite will react as a monolithic glass pane. This is nearly suitable for a dynamic load application as time-dependent properties of the interlayer material are less significant. Regarding the other limiting case of an ideally flexible interlayer material, the panes respond independently. Flexibility in shear of the interlayer reduces the transmission of horizontal shear stresses, so that the single glass panes are less capable to interact with each other and the deflection increases. Viscous material behavior of the interlayer comes to the fore in static loading.

\subsection{Damage configurations}

Due to its residual load carrying capacity, the failure of laminated safety glass occurs progressively. Even under a high dynamic blast loading the composite is able to pass through damage configurations depending on the blast characteristics.

The post-breakage behavior of laminated safety glass for static load cases has been studied by various research groups. Seshadri et al. (2002) introduce an analytical model and a simulation technique for cracked laminates. Kott and Vogel (2006) investigate the residual load carrying capacity after fracture experimentally. The performance of laminated safety glass 
Fig. 1 Failure mechanisms in laminated safety glass and load transfer on alternative mechanically carrying paths

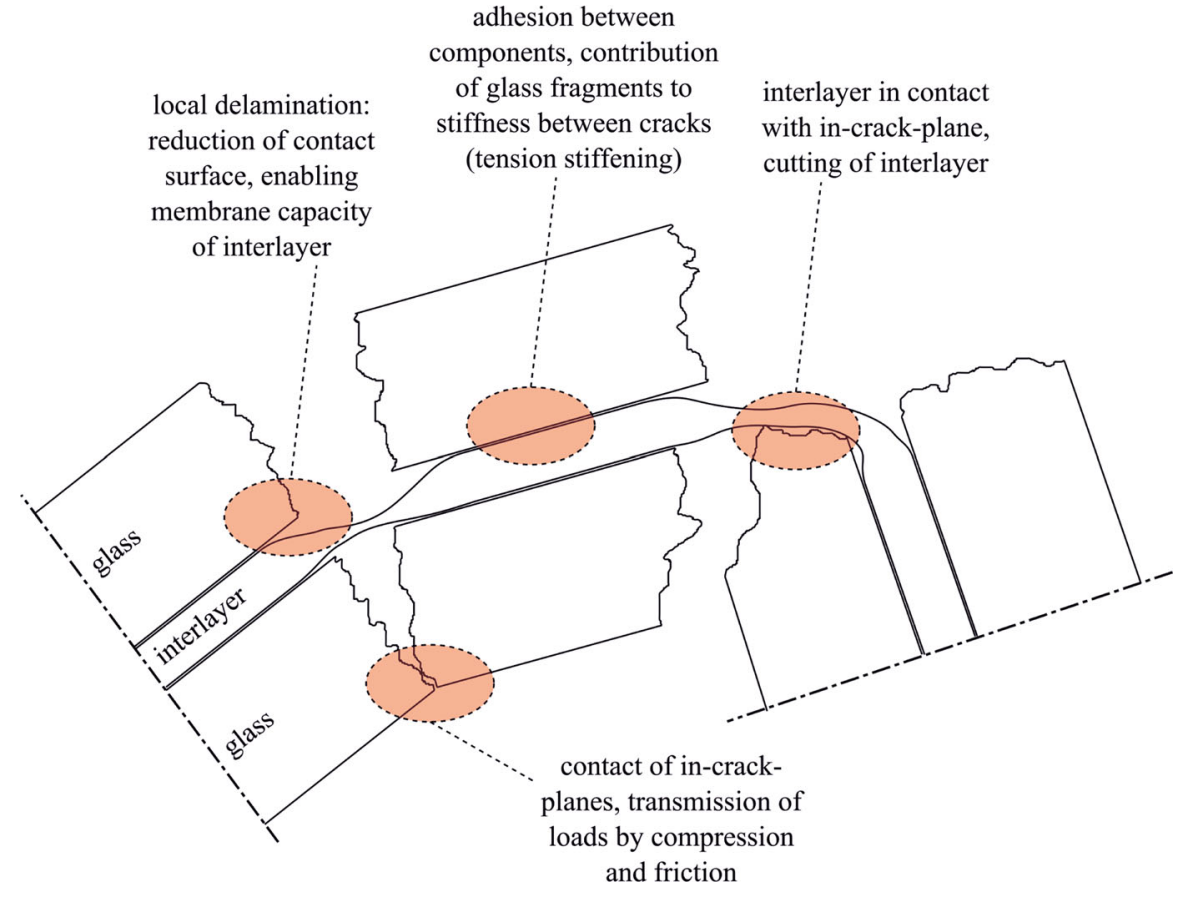

adhesion between mponents, contribution of glass fragments to tiffness between cracks with in-crack-plane, cutting of interlaye under blast beyond the elastic state has been examined in detail by Kuntsche (2015), Palermo and Liu (2015), Zhang (2014) and Morison (2010).

In the first instance, one of the outer single glass panes fails, which leads to a sudden reduction of flexural stiffness. Two cases are distinguished. If the overpressure history of an explosion is driven by the positive impulse, the laminate will be able to respond in a combined bending and membrane mode. The single glass pane on the protected side is set under higher tension and fails firstly due to the low tensile strength of glass. Following deflection is coupled with the failure of further glass plies in the tensile section. The stiffness decreases gradually.

After the cracking of glass, a majority of fragments sticks to the interlayer. This enables the laminate to transfer loads on alternative mechanically carrying paths. Figure 1 demonstrates the failure mechanisms, which might occur while loaded. Alike the tension stiffening in cracked reinforced concrete, stresses are induced in adherent glass fragments, so that they contribute to the stiffness between cracks. In addition, a transmission of stresses takes place in-crack-plane by friction and compression.

In close proximity to a crack the interlayer starts delaminating from the glass surface. The more pronounced the local delamination occurs, the more increases the initial length of the free interlayer between the delamination fronts. In consequence the straining of the interlayer is mitigated. The structural behavior of the fractured composite is increasingly governed by the membrane mode. Ultimately, the interlayer fails by reaching its tensile failure strain or is cut when being in contact with sharp glass fragments.

If the overpressure history is dominated by the maximum reflected overpressure, the ductility of the composite structure will eventually not be exploited. In this case of loading, the single glass ply on the attacked side may fail straightaway due to the impact of the blast wave. The loading enhances the likeliness of a sudden failure by dynamic shear in the area close to the bearing. Stages of progressive failure described previously are less pronounced. The described cases of structural response are shown in Figure 2a, b respectively. Figure $2 \mathrm{c}$ illustrates laminate fragments remaining in the frame construction after shear failure.

\subsection{Work balance}

The laminate performance beyond cracking is assessed on the basis of a work balance. In shock tube testing, the reflected overpressure-time history $\mathrm{p}_{1, r}(\mathrm{t})$ characterizes the blast loading. Several approaches for the determination of blast parameters can be found in lit- 
Fig. 2 a and b Different structural responses of laminated safety glass under blast loading, extensive development of a membrane (a), failure by dynamic shear near the boundary (b), laminate fragments remaining in frame construction after shear failure (c), courtesy of WTD 52

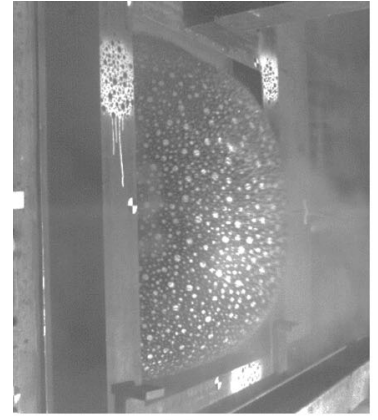

(a)

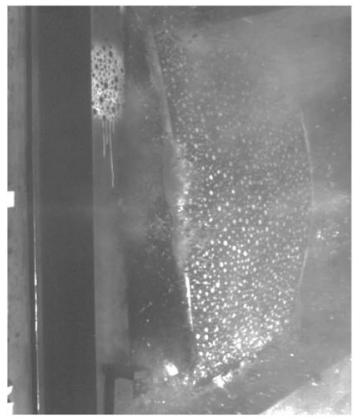

(b)

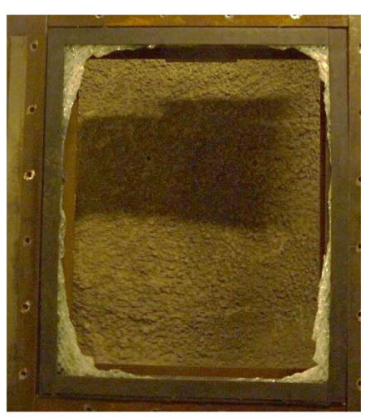

(c) erature (Henrych and Major 1979; Korenev and Rabinovič 1985; Kinney and Graham 1985). $\mathrm{p}_{1, \mathrm{r}}(\mathrm{t})$ can be described i.e. by a linearly decreasing function or polynomial functions of higher orders. In the following, $\mathrm{p}_{1, \mathrm{r}}(\mathrm{t})$ is approximated with sufficient accuracy by the Friedlander equation $\mathrm{p}_{\text {Friedlander }}(\mathrm{t})$ (Friedlander 1939).

$p_{\text {Friedlander }}(t)=p_{01, r} \cdot\left(1-\frac{t}{t_{+}}\right) \cdot e^{-b \cdot\left(\frac{t}{t_{+}}\right)} \approx p_{1, r}(t)$

With known reflected peak overpressure $\mathrm{p}_{01, \mathrm{r}}$ and duration of the overpressure phase $t_{+}$, the Friedlander parameter $b$ is determined through curve fitting. The plane surface of the initial state and the curved surfaces in any actual configuration encase a certain space, $V_{\text {enc }}$. The encased volume-time history is evaluated for the time of structural response until the encased volume reaches its maximum peak or an opening occurs in the laminate. It is assumed, that the superposition of $\mathrm{p}_{\text {Friedlander }}(\mathrm{t})$ and the time derivative of $\mathrm{V}_{\text {enc }}(\mathrm{t})$ captures the main part of external work executed on a laminated safety glass specimen.

$W_{\text {ex }} \approx \int \dot{V}_{\text {enc }}(t) p_{\text {Friedlander }}(t) d t$

The external work correlates with the internal work as stored deformation energy and the energy released in dissipation processes. In a first step, the internal work is split up for elastic and damaged configurations.

$W_{e x}=W_{i n}=W_{i n, e l}+W_{i n, d m g}$

The first occurrence of glass fracture confines the elastic state of the laminate in time. It is assumed, that $\mathrm{W}_{\mathrm{in}, \mathrm{el}}$ equals the stored energy of elastic deflection $E_{\text {defl,el }}$ in the bending and membrane mode
$W_{i n, e l}=E_{d e f l, e l}$

and $\mathrm{W}_{\mathrm{in}, \mathrm{dmg}}$ yields the summation of glass fracture energy $\mathrm{E}_{\text {frac }}$, local delamination $\mathrm{E}_{\text {delam }}$ and interlayer deformation $\mathrm{E}_{\mathrm{def}}$.

$W_{\text {in,dmg }}=E_{\text {frac }}+E_{\text {delam }}+E_{\text {def }}$

Further energy portions, i.e. friction in-crack-plane or the heating of the interlayer due to the high strain rates, are neglected at this point. Follow-on investigations will be required to determine, if other energy losses can be assumed of minor importance. The elastic deflection of the laminate is limited by the practical tensile strength of the glass plies. The dynamic flexural stiffness of the composite cross section governs the extent of deflection. Crack spacing restricts the potential of local delamination and interlayer deformation, so that the portions of $E_{\text {frac }}, E_{\text {delam }}$ and $E_{\text {def }}$ interdepend. After occurrence of cracking, the fractions $\mathrm{W}_{\text {delam }}$ and $\mathrm{W}_{\text {def }}$ are assumed to contribute significantly to the performance of the composite.

The paper aims at the determination of $\mathrm{W}_{\text {in,el }}$ and $\mathrm{W}_{\text {in,dmg }}$ and the establishment of interrelations between $E_{\text {frac }}, E_{\text {delam }}$ and $E_{\text {def }}$. As $E_{\text {frac }}$ can be derived from the fracture pattern, the ratio of $E_{\text {delam }}$ to $E_{\text {def }}$ remains as sole unknown.

\subsection{Energy of local delamination}

Since the "peel test" and the "pummel test" are not entirely appropriate to determine the fracturemechanical characteristics of the composite interfaces, i.e. in the case of highly ductile interlayers, Sha et al. (1997) developed the test set-up of through-crackedtensile-testing (TCT-testing). The test-set-up repre- 
sents uniaxial tensile testing of laminated glass with a defined crack in the glass plies. Measured loaddisplacement and delamination-displacement histories are used to determine the critical energy release rate $\Gamma_{\mathrm{c}}$ with given or reliably assumed material properties of the interlayer. This fracture mechanical value describes the energy required for the creation of crack surfaces. In case of load application in TCT-testing, the specified value is representative for a combined mode I-II fracture. $E_{\text {delam }}$ is obtained from the integration of $\Gamma_{\mathrm{c}}$ over the delaminated surface.

Several research groups investigate the interface properties of laminated safety glass with different emphasis. Iwasaki et al. (2007), Feretti et al. (2012) as well as Butchart and Overend (2012) conducted TCTtesting under variation of the haul-off speed. $E_{\text {delam }}$ increases for higher loading velocities. On the contrary, $\mathrm{E}_{\mathrm{def}}$ remains of the same magnitude due to energetic neutralization of stiffening and decreasing deformation of the interlayer. The rate dependence of the fracture process has been investigated by Muralidhar et al. (2000) for long and short delamination lengths. Franz et al. (2014) investigated the delamination behavior among interlayers with varying adhesion grades.

\subsection{Energy of interlayer deformation}

Most common interlayers used for lamination with glass are polymeric materials. This material group is able to sustain large deformations. The material behavior shows high sensitivity to the strain rate and is strongly dependent on temperature in the conventional application range of civil engineering. Williams et al. (1955) capture the interrelationship between the straining frequency, temperature and material properties in the principle of the frequency-temperature shift.

Looking at the macromolecular structure of polymers, the phenomenological behavior can be explained mechanically according to Schwarzl (1990). Important characteristics, that determine the reaction of the molecular network to loading, are the density of interlacing and entanglements, crystallinity as well as the polymerization degree. The constitutive material models developed by Arruda and Boyce (1993) and Boyce et al. (2000) account for the macromolecular structure.

An amorphous polymer and amorphous domains of partially crystalline polymers exist with an increasing temperature in a glassy, viscous or molten aggregate state. When reaching the glass transition temperature, the energy barrier to enable molecular chain movements is exceeded. The glass transition temperature may vary severely for different types of polymers. A substantial change in the behavior of the material can be observed, as the polymer turns from a glassy state into a viscous state. This has an effect on the breakdown of the total strain in elastic, plastic and viscous portions. Apparent plastic deformations are entropy-elastic, as they can be reversed by warming. The phenomenon is denoted as thermal spring-back.

\section{Experimental investigation}

\subsection{Test set-up}

In the first quarter of 2015 shock tube testing of laminated glass panels has been conducted at the facilities of the WTD 52 in Oberjettenberg. The normative glazing standard EN 13541 (2012) for testing and classification of the resistance against explosion pressure provides the basis for the test set-up. The diameter of the shock tube amounts $2 \mathrm{~m}$. In first instance, the attention must be centered on the reproducibility of boundary conditions, as the bearing is responsible for the in-field response of the laminate. The framing construction is depicted in Fig. 3 as schematic drawing. Steel sheets fixed by circumferentially arranged bolted connections ensure an all-side rigid clamping of the specimen. The bite length is an important factor regarding the pull-out of the specimen. There it is chosen to be $50 \mathrm{~mm}$ according to Fig. 3. The strength of clamping is defined by the bolts spaced in distance of $20 \mathrm{~cm}$ and a torque moment of $12.5 \mathrm{Nm}$. Viscous effects of the isolation material between the specimen and the framing may distort the clamping conditions. A natural rubber with a characteristic Shore-A value range of $65 \pm 7$ is applied as isolation material. The torque moment is applied immediately before the safety buffer of the explosion.

The kinematic variables of the specimen are determined through Digital Image Correlation (DIC). For the purpose of 3D deformation measurement, two pairs of high speed video cameras are installed. The second pair is thereby used for redundant recording of the structural response and independent controlling of the measurements. The camera positions are shown in Fig. 4b. The protected side of the specimen is initially 
Fig. 3 Schematic drawing of the framing construction, all dimensions given in $\mathrm{mm}$
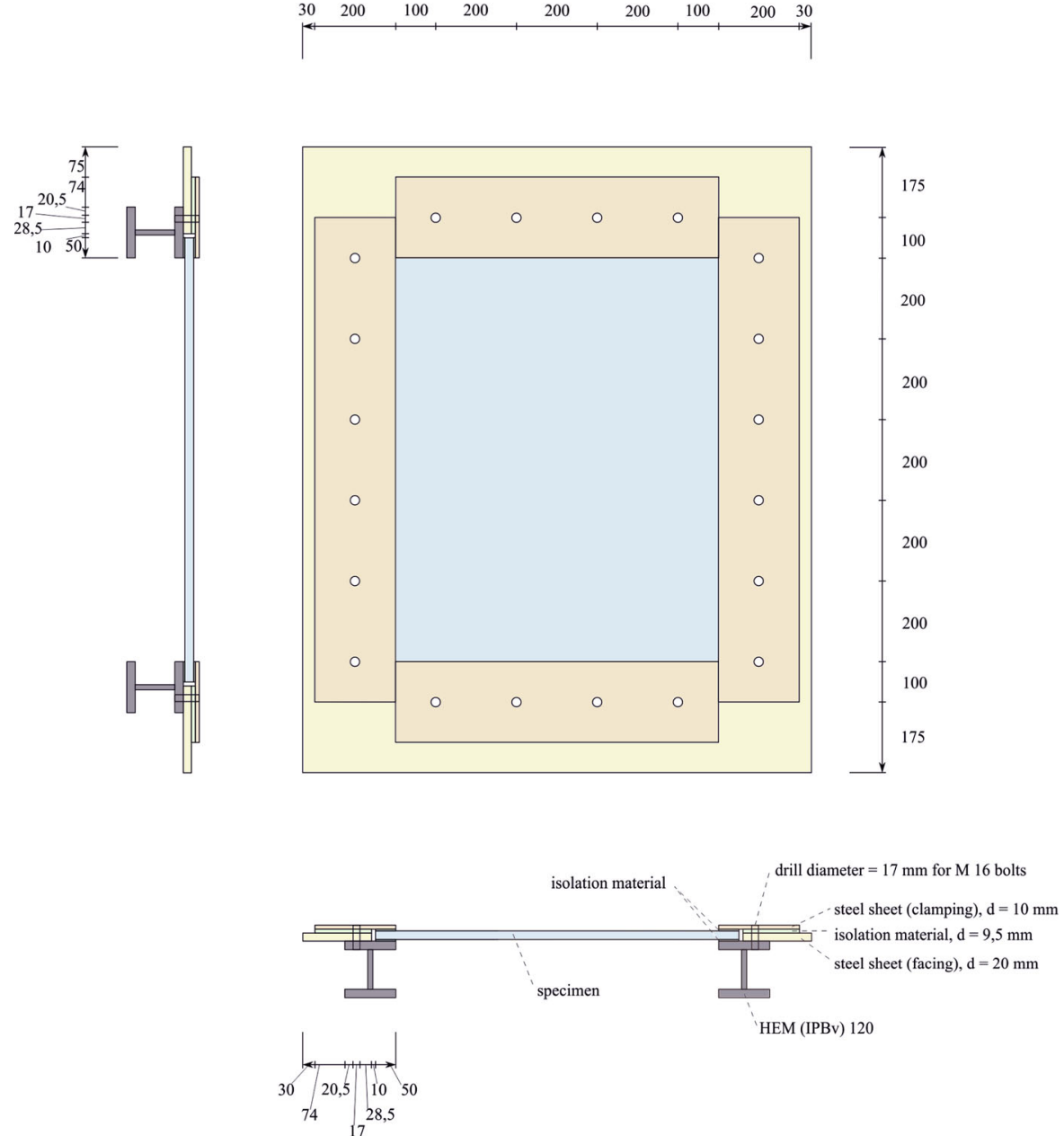

HEM (IPBv) 120

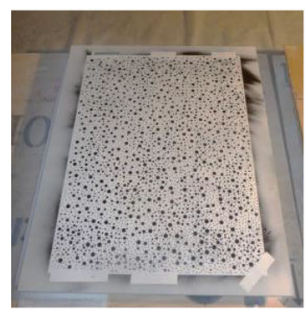

(a)

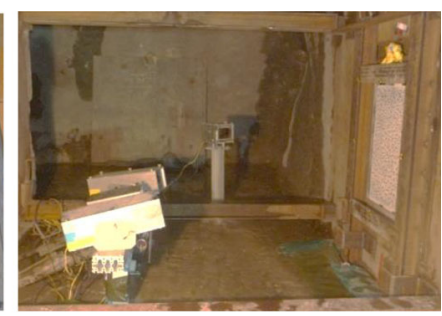

(b)
Fig. 4 a and $\mathbf{b}$ Preparation of the speckle pattern on protected specimen surface (a), camera set-up on protected side (b), courtesy of WTD 52

surfaced with a speckle pattern. As the unaffected fracture process on the protected surface is of particular interest, the pattern is not brought up by the use of a film but by varnishing. The speckle sizes are harmonized with the distance between the cameras and the specimen. Figure 4 a depicts the varnished specimen in preparation for the test. The comparison of the pattern states at different points in time enables optical contactless measurement of spatial deformation patterns on a curved surface. Furthermore, piezoelectric pressure transducers deliver the reflected overpressure-time histories on the attacked side of the specimen. In addition, the surface temperature of the specimen as well as the ambient temperature and air humidity are recorded before triggering the explosion.

\subsection{Fabrication of specimens}

All specimens are $1100 \mathrm{~mm}$ long and $900 \mathrm{~mm}$ wide. They are all equal in their symmetrical cross-sectional buildup $10 \mathrm{~mm}$ glass - interlayer-10 mm glass with the single pane thickness given as nominal thickness. The real thickness of a single pane amounts $9.80 \mathrm{~mm}$. 
Table 1 Specimen configurations

\begin{tabular}{llllr}
\hline Specimen label & Glass type & Interlayer & Lamination process & Quantity \\
\hline F-P-a & Annealed & Trosifol BG R20 (PVB) & Autoclave & 10 \\
F-Pr-a & Annealed & Trosifol BG R10 (PVB) & Autoclave & 7 \\
F-Pv-v & Annealed & Trosifol HR 100 (PVB) & Vacuum & 7 \\
F-T-a & Annealed & covestro Dureflex A4700 (TPU) & Autoclave & 2 \\
F-E-a & Annealed & Bridgestone EVAsafe & Autoclave & 2 \\
F-I-a & Annealed & Kuraray SentryGlas & Autoclave & 4 \\
F-ES-a & Annealed & Trosifol ES (PVB) & Autoclave & 6 \\
T-Pr-a & Heat strengthened & Trosifol BG R10 (PVB) & Autoclave & 3 \\
E-Pr-a & Fully tempered & Trosifol BG R10 (PVB) & Autoclave & 3 \\
\hline
\end{tabular}

Table 2 Selected material properties of applied interlayers (glass transition temperature based on work by Schuster (2014))

\begin{tabular}{|c|c|c|c|c|c|}
\hline Interlayer & $\begin{array}{l}\text { Shear stiffness } \\
G(t, T) \text { (reference) }\end{array}$ & $\begin{array}{l}\mathrm{G}(\mathrm{t}=3 \mathrm{~s} \\
\left.\mathrm{T}=30^{\circ} \mathrm{C}\right)[\mathrm{MPa}]\end{array}$ & $\begin{array}{l}\text { Tensile strength } \\
\text { ISO } 527[\mathrm{MPa}]\end{array}$ & $\begin{array}{l}\text { Tensile strain ISO } \\
527[\%]\end{array}$ & $\begin{array}{l}\text { Glass transition } \\
\text { temperature }\left[{ }^{\circ} \mathrm{C}\right]\end{array}$ \\
\hline Trosifol BG R20 & $*$ & ca. 0.5 & $>23$ & $>280$ & ca. 30 \\
\hline Trosifol BG R10 & $*$ & ca. 0.5 & $>23$ & $>280$ & ca. 30 \\
\hline Trosifol HR 100 & $*$ & ca. 0.5 & $>20$ & $>250$ & ca. 30 \\
\hline Dureflex A4700 & - & - & $>37$ & $>500$ & ca. -40 \\
\hline Bridgestone EVAsafe G77 & Goebel (2013) & 3 & $>10$ & $>500$ & ca. -16 \\
\hline Kuraray SentryGlas & SentryGlas (2015) & 141 & $>34.5$ & $>400$ & ca. 53 \\
\hline Trosifol ExtraStrong & Kuraray (2015) & 58 & $>32$ & $>180$ & ca. 41 \\
\hline
\end{tabular}

* PVB shear stiffness is discussed in many different publications, for further details we refer to Sobek et al. (2000), Schuler (2003), Ensslen (2005), Sackmann (2008), and COST (2013)

The interlayer thickness before lamination is about $2.28 \mathrm{~mm}$ depending on the available single interlayer thickness. The interlayer thickness after lamination is approximately $1.90 \mathrm{~mm}$. The specimens differ in tempering of the glass plies, interlayer material and lamination process. The configurations, the quantities and the labels of the specimens are summarized in Table 1.

Most of the specimens are laminated with float glass. In order to assess the influence of glass strength, some specimens with the PVB interlayer Trosifol BG R10 are also fabricated with heat strengthened and fully tempered glass. The characteristic tensile bending strength of annealed glass is $45 \mathrm{MPa}$, of heat strengthened glass 70 and $120 \mathrm{MPa}$ for fully tempered glass. In shock tube testing, these values may be enhanced by the strain-rate-effect. Since the glass tensile strength highly depends on surface damage and micro cracks, all glass plies are treated with abrasive paper to mini- mize strength scattering. In consequence, the expected glass tensile strength is assumed to be close to the characteristic value.

The stiffness of laminated glass is determined by the thickness of its components, the Young's modulus of glass (70-75 GPa), and the interlayer shear stiffness $\mathrm{G}$, which determines the composite action of the laminate. The blast performance of the laminated glass is also influenced by the adhesion of the interlayer to the glass and the interlayer elongation at failure.

Table 2 compares some selected basic material properties of the used interlayers for quasi-static load application at moderate temperatures. It must be stated, that these characteristics may vary significantly in comparison to those measured under dynamic loading, i.e. the stiffness of the interlayer tends to increase at higher strain rates. All Trosifol interlayers are PVB films which differ in adhesion strength, shear stiffness and processing technique. BG R10 provides a medium 
adhesion for laminated glass. BR R20 has a higher adhesion than R10. Both, BG R10 and R20, have a tensile strength of at least $23 \mathrm{MPa}$ and a tensile elongation of more than $280 \%$ (based on ISO 527 testing). In contrast to BG R10 / R20, the PVB film HR 100 can also be processed without any autoclave by applying a vacuum for 12 hours and a temperature of not more than $30{ }^{\circ} \mathrm{C}$. The tensile strength is $>20 \mathrm{MPa}$ and the tensile elongation $>250 \%$.

Besides the "classical" PVB films, the authors also looked at structural PVB (Extra Strong ES), Polyurethane (TPU), EVA and SentryGlas interlayers. EVA can be processed with methods other than autoclaving (e.g. vacuum laminator), but the EVA specimens for the present test series were laminated in the autoclave. Both PVB ES and SentryGlas are much stiffer and stronger than normal PVB films and are often used for structural glass applications. TPU interlayers are developed for the lamination of glass and polycarbonate. Acrylic composites and other compatible thermoplastics are also laminated with TPU interlayers.

\subsection{Preliminary assessment of thermal behavior}

The heating and cooling process of the tested specimen type has been studied and optimized in a reference test with a heat transition analysis beforehand. The surface temperature of the attacked side is measured at nine gauge points distributed across the surface. Figure 5 shows exemplarily the history of measured surface temperature at the center gauge point on the attacked side of the specimen. The heating process begins with a cold start of a heater blower, which applies a temperature load unilaterally onto the protected side of the specimen. After approximately one hour of heating, the temperature increments on the surface decline. This implies the approach to a stationary heat transfer. Further heating beyond this point in time is inefficient, so that the duration of the heating process is set to $3720 \mathrm{~s}$. In addition, this method ensures, that the core of the laminate is heated and the temperature profile across the thickness is sufficiently defined at the end of the heating process.

Any auxiliary attachments are removed for an undisturbed cooling. For this process, measurements of the surface temperature are taken at the same gauge points but from both the protected and the attacked side. The

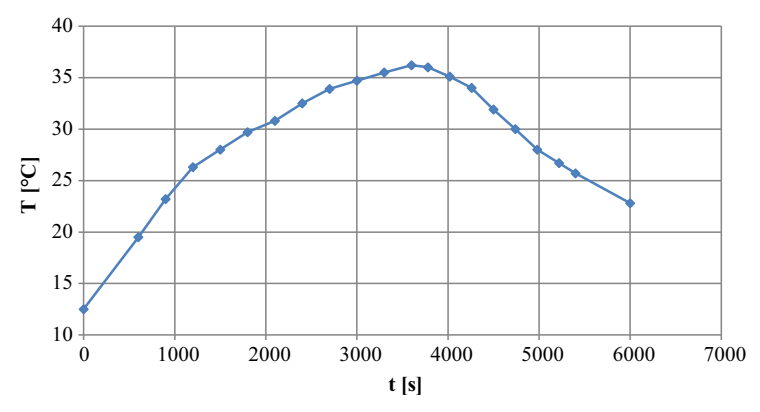

Fig. 5 Measured surface temperature at the center gauge point on attacked side of the specimen after a heating period of $3720 \mathrm{~s}$

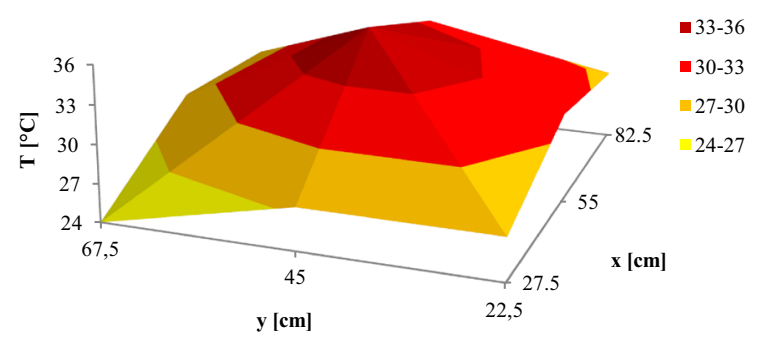

Fig. 6 Surface temperature state on attacked specimen side after a heating period of $3720 \mathrm{~s}$, linear interpolation between gauge points

surface temperature state on the attacked side of the specimen after the heating period is captured in Fig. 6. The temperature varies across the surface. The temperature of the edge region is lower than in the center area, which has been directly exposed to the air stream of the heater blower. The temperature state of the surfaces is monitored for another half an hour of cooling to determine the cooling behavior of the heated specimen at ambient temperature.

With the specific thermal conductivity of glass and a typical interlayer, the temperature on the component interfaces can be determined, as the heat transfer is stationary at the beginning of cooling. It is assumed, that the core temperature remains higher than the surface temperatures after some cooling. In nearly any of the tests the detonation could be triggered not later than $240 \mathrm{~s}$ after termination of the heating process. Due to the marginal decrease of surface temperatures during this period, the cooling of the interlayer in the core of the laminate is neglected.

It is defined, that the mean value of the throughthickness interlayer temperature gradient represents the interlayer temperature at a gauge point. The in-plane 
Table 3 Characteristic temperatures describing the in-plane temperature state of the interlayer at the time of detonation

\begin{tabular}{llc}
\hline $\mathrm{T}_{\text {int,max }}\left[{ }^{\circ} \mathrm{C}\right]$ & $\mathrm{T}_{\text {int,min }}\left[{ }^{\circ} \mathrm{C}\right]$ & $\mathrm{T}_{\text {int,mean }}\left[{ }^{\circ} \mathrm{C}\right]$ \\
\hline 36.2 & 24.2 & 30.0
\end{tabular}

Table 4 Configurations of combined blast-temperature loading

\begin{tabular}{llllll}
\hline Loading label & \multicolumn{2}{l}{ Air blast load } & & $\frac{\text { Temperature load }}{n}$ \\
\cline { 2 - 4 } & $\mathrm{p}_{01, \mathrm{r}}[\mathrm{kPa}]$ & $\mathrm{i}_{+, \mathrm{r}}[\mathrm{Pa} \mathrm{s}]$ & $\mathrm{t}_{+}[\mathrm{ms}]$ & $\mathrm{b}[-]$ & \\
\hline $1-1$ & 95 & 1100 & 45 & 1.5945 & 13 \\
$1-2$ & 95 & 1100 & 45 & 1.5945 & 30 \\
$2-1$ & 170 & 1100 & 28 & 3.0532 & 13 \\
$2-2$ & 170 & 1100 & 28 & 3.0532 & 30 \\
$3-1$ & 225 & 2300 & 25 & 2.2821 & 13 \\
$3-2$ & 225 & 2300 & 25 & 2.2821 & 30 \\
\hline
\end{tabular}

temperature state of the interlayer after repetition of the defined heating process at the time of detonation is then characterized by the maximum temperature $\mathrm{T}_{\text {int,max }}$, the minimum temperature $T_{i n t, m i n}$ and the mean temperature $T_{\text {int,mean }}$ of temperatures measured at nine gauge points in the reference test. These characteristic temperatures are shown in Table 3.

\subsection{Execution, testing schedule and specimen performance}

The laminate is exposed to a combination of blast and temperature loading. The load configurations are presented in Table 4. The air blast wave in a shock tube is characterized by the reflected peak overpressure $\mathrm{p}_{01, \mathrm{r} \text {, }}$ the reflected positive impulse $i_{+, r}$ and the duration of the overpressure phase $t_{+}$. In addition to the blast parameters, the Friedlander parameter $\mathrm{b}$ is given for the approximation of measured reflected overpressuretime history. The lowest loading configuration is chosen to achieve an elastic response of all tested specimens and corresponds to the requirements of the blast resistance class ER 1 (EN 13541 2012). For the upper bound of the loading parameters, total failure or serious damage of the specimens is expected. The highest blast loading configuration accords with ER 4. In a preliminary investigation, the loading increments have been set to be within the ranges of different damage states for the intermediate region. The medium blast load level complies with ER 2.
The laminate is tested at two temperature levels. The temperature is thereby related to $\mathrm{T}_{\text {inter,mean }}$. The ambient conditions in the testing facility are constant at a temperature of $13{ }^{\circ} \mathrm{C}$ and an air humidity of $70 \%$. They represent the storage conditions as well as the lower temperature level. For the elevated temperature level, the temperature load is applied as mentioned in the previous section. The temperature state of the specimen is monitored at random to confirm being within the determined heating process. Then auxiliary attachments are removed and the detonation is triggered after the safety buffer.

A single test is subsequently abbreviated by the identifier serial number - specimen label - loading label. The testing schedule and the overall performance of the specimen are summarized in Table 5 for a selection of tests. The fracture of glass is accompanied by an, in part dramatic, secondary debris hazard.

\section{Discussion of results}

In the following, the evaluation focusses on the influence of the loading configuration, the influence of different interlayer materials and the influence of glass tempering.

\subsection{Center displacement during elastic response}

In the first instance, the test series with an exclusively elastic response are discussed. Figure 7 depicts the cen- 
Table 5 Extract of testing schedule and overall performance of the specimens

\begin{tabular}{ll}
\hline Identifier & Overall performance of specimen \\
\hline 4-F-Pr-a-1-1 & Elastic response \\
11-F-Pr-a-1-2 & Fracture of both glass plies, intact interlayer \\
13-F-I-a-1-2 & Elastic response \\
14-E-Pr-a-1-2 & Elastic response \\
19-F-Pr-a-2-1 & Fracture of both glass plies, intact interlayer \\
20-F-Pv-v-2-1 & Fracture of both glass plies, tearing of interlayer from center \\
21-F-P-a-2-2 & Fracture of both glass plies, tearing of interlayer from center \\
23-F-Pr-a-2-2 & Fracture of both glass plies, intact interlayer \\
24-F-Pv-v-2-2 & Fracture of both glass plies, tearing of interlayer from center \\
25-F-I-a-2-2 & Fracture of both glass plies, intact interlayer \\
26-E-Pr-a-2-2 & Fracture of glass ply on protected side only \\
27-T-Pr-a-2-2 & Fracture of both glass plies, intact interlayer \\
28-F-ES-a-2-2 & Fracture of both glass plies, circumferential tearing of interlayer near boundary \\
31-F-Pr-a-3-1 & Fracture of both glass plies, circumferential tearing of interlayer near boundary \\
33-F-T-a-2-2 & Fracture of both glass plies, tearing of interlayer from center \\
34-F-E-a-2-2 & Fracture of both glass plies, tearing of interlayer from center \\
36-F-Pr-a-3-2 & Fracture of both glass plies, tearing of interlayer from center \\
39-E-Pr-a-3-2 & Fracture of both glass plies, circumferential tearing of interlayer near boundary \\
40-T-Pr-a-3-2 & Fracture of both glass plies, circumferential tearing of interlayer near boundary \\
\hline
\end{tabular}

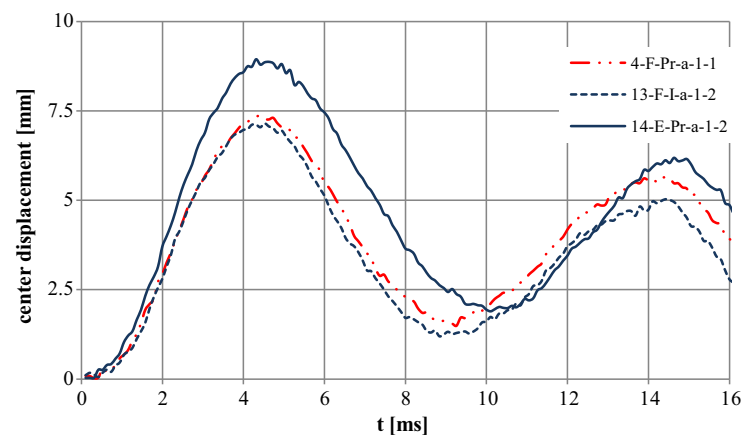

Fig. 7 Center displacement versus time, different specimens with elastic response at low blast loading

ter displacement of different specimens over time for testing under a low blast load. The specimen of test 4 , with a PVB interlayer tested at low temperature, shows approximately the same frequency and amplitude of the oscillation as the specimen of test 13 with an ionoplast interlayer at elevated temperature. The specimen configuration F-Pr-a did not remain elastic at elevated temperature level. The specimen of test 14 is tested at elevated temperature and owns the same interlayer. It is laminated with tempered glass plies, which enhances the resistance against early glass fracture through a more intense deflection. The history shows increased amplitudes and a lower frequency, inducing a decreased flexural stiffness of the composite due to the presumable thermal softening of the PVB interlayer at elevated temperature. With regard to the interlayer temperatures in considered tests, the elastic response of the laminate may be influenced by approaching or partially exceeding the glass transition.

\subsection{Investigation of the fracture pattern}

Based on the fracture pattern, the potential of energy dissipation can be quantified for the considered specimen. A high density of cracks induces an increased possibility of dissipation by the mentioned mechanisms illustrated in Fig. 1. Further investigation and establishing a relation to dissipated energy portions will show, whether this potential has been exploited by the composite.

Figure 8a depicts the set-up for photographic recording of the fracture pattern. The damaged specimen is removed carefully from the frame support and placed with the protected surface upside on a transparent table, 


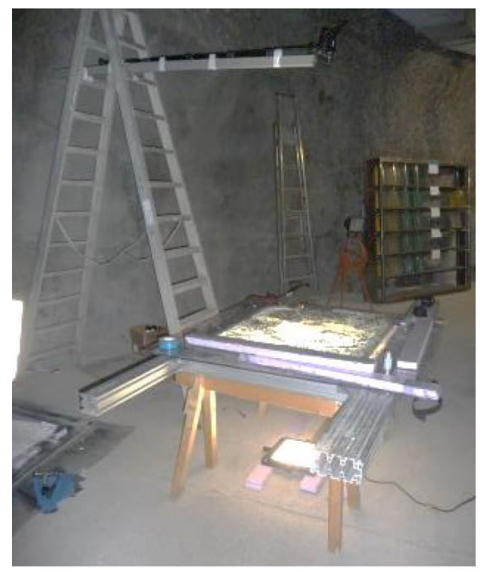

(a)

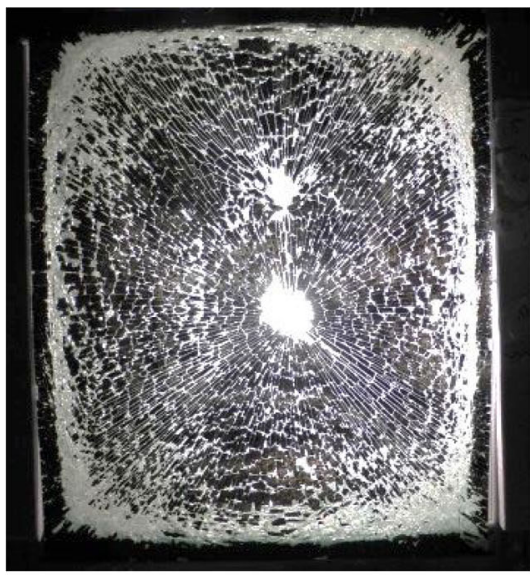

(b)

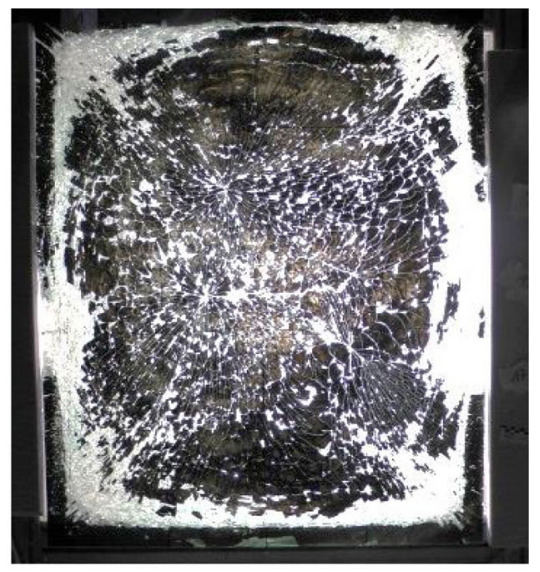

(c)

Fig. 8 a, b and c Set-up for photographic recording of the fracture pattern (a), fracture patters of tests 23-F-Pr-a-2-2 (b) and 20-F-Pvv-2-1 (c), courtesy of WTD 52

which is illuminated from below. The fracture pattern evolved through the varnish coat of the speckle pattern and is now visible, as the light shines directly through the cracks, whereas uncracked areas remain dark. A photograph is taken from the protected side of the specimen out of a long shot. Examples of fracture patterns highlighted in such a way are shown in Fig. 8b, c.

On the one hand the fracture patterns own similarities: Near the rigid support, the glass fragments get in contact, which leads to a cohesion failure by shear and a break-out of debris. This becomes evident by the bright areas forming an outside ring near the clamping. Fracture lines cross the surface diagonally from the corners through the field as a result of the main bending moment distribution. On the other hand the patterns differ in their appearance. In Fig. $8 \mathrm{~b}$ two bright, major spots in the field indicate extensive areas of adhesion failure between the components. There is no opening, but solely the pure interlayer. These spots represent the centers of a regular cobweb-like crack pattern. However, the pattern illustrated in Fig. 8c is more diffuse and owns numerous crack centers. A clear orientation of fracture lines cannot be observed from this perspective.

In a next step, the fracture patterns are described statistically. The clear surface on the protected side is subdivided into twenty sectors as shown in Fig. 9a. Each sector, corresponding to a square with a side length of $20 \mathrm{~cm}$, is photographed as close-up. Due to the influence of the support on the fracture behavior near the clamping area, only the field sectors (marked in red) are considered. In the field, the pattern mainly evolved in tension and shows a directed and regular crack distribution, which is depicted in Fig. 9b. The crack edges are clearly separated and some fragments are separated from the surface.

Starting from a crack center, the fracture lines are orientated tangentially and radially. 10 paths of $10 \mathrm{~cm}$ arc length (five per orientation) are fitted into a sector image, respectively. They should intersect the fracture lines in a right angle and be smoothly distributed across the sector. Furthermore, the paths should not run across discontinuities as laminate openings or areas of extensive adhesion failure to capture the global crack pattern. The number of cracks intersecting a path is determined as crack density. The fracture pattern in the field can then be characterized by

- the mean number of tangential cracks along a path length of $10 \mathrm{~cm} \mathrm{n}_{\mathrm{cr}, \text { tang }}$ and its standard deviation $\sigma_{\mathrm{cr}, \text { tang, }}$,

- the mean number of radial cracks along a path length of $10 \mathrm{~cm} \mathrm{n} \mathrm{n}_{\mathrm{cr}, \mathrm{rad}}$ and its standard deviation $\sigma_{\mathrm{cr}, \mathrm{rad}}$ and

- the number of crack centers $n_{\text {crc }}$.

The comparison of tests with the same loading and specimen configuration does not result in a major scattering of crack densities. Table 6 shows the characteristics for the testing of F-Pr-a configurations. It is found, that $\mathrm{n}_{\mathrm{cr} \text {, tang }}$ and $\mathrm{n}_{\mathrm{cr}, \text { rad }}$ decrease with increasing temperature load. However, the blast load level has a low influence on the evaluated crack densities. The coeffi- 
Fig. 9 a and b Partitioning of the clear specimen surface in sectors (a), close-up of a field sector with paths intersecting tangential (red) and radial (green) cracks. (Color figure online)

\begin{tabular}{|c|c|c|c|}
\hline 1 & 2 & 3 & 4 \\
\hline 5 & 6 & 7 & 8 \\
\hline 9 & 10 & 11 & 12 \\
\hline 13 & 14 & 15 & 16 \\
\hline 17 & 18 & 19 & 20 \\
\hline
\end{tabular}

subsumed sectors for further statistical evaluation

(a)

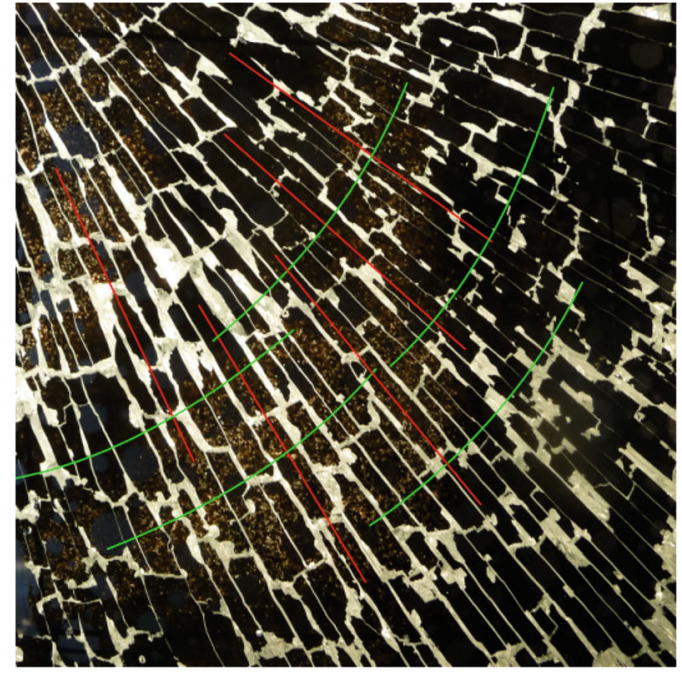

(b)

Table 6 Characteristics of the fracture pattern, test series F-Pr-a at different loading configurations

\begin{tabular}{llllll}
\hline Identifier & $\mathrm{n}_{\text {cr,tang }}[1 / 10 \mathrm{~cm}]$ & $\sigma_{\text {cr, tang }}$ & $\mathrm{n}_{\text {cr,rad }}[1 / 10 \mathrm{~cm}]$ & $\sigma_{\text {cr, rad }}$ & $\mathrm{n}_{\text {crc }}$ \\
\hline 11-F-Pr-a-1-2 & 4.0 & 1.0 & 15.1 & 2.7 & 1 \\
19-F-Pr-a-2-1 & 5.1 & 1.5 & 18.2 & 2.6 & 1 \\
23-F-Pr-a-2-2 & 3.9 & 1.2 & 14.7 & 2.3 & 2 \\
31-F-Pr-a-3-1 & 6.2 & 1.6 & 20.4 & 2.8 & 2 \\
36-F-Pr-a-3-2 & 5.1 & 1.6 & 15.1 & 2.1 & 1 \\
\hline
\end{tabular}

Table 7 Characteristics of the fracture pattern, different specimen configurations at medium blast load and elevated temperature

\begin{tabular}{lcllll}
\hline Identifier & $\mathrm{n}_{\text {cr, tang }}[1 / 10 \mathrm{~cm}]$ & $\sigma_{\text {cr, tang }}$ & $\mathrm{n}_{\text {cr, rad }}[1 / 10 \mathrm{~cm}]$ & $\sigma_{\text {cr, rad }}$ & $\mathrm{n}_{\text {crc }}$ \\
\hline 21-F-P-a-2-2 & 4.0 & 1.3 & 13.2 & 3.6 & 2 \\
37-F-Pv-v-2-2 & 5.2 & 2.1 & 13.7 & 3.9 & 2 \\
25-F-I-a-2-2 & 6.3 & 1.4 & 14.9 & 2.3 & 1 \\
28-F-ES-a-2-2 & 6.5 & 1.6 & 15.8 & 3.3 & 1 \\
33-F-T-a-2-2 & 4.2 & 1.4 & 13.3 & 2.6 & 2 \\
34-F-E-a-2-2 & 5.9 & 1.4 & 13.5 & 3.0 & 1 \\
26-E-Pr-a-2-2 & 11.2 & 2.5 & 31.9 & 3.4 & 4 \\
27-T-Pr-a-2-2 & 5.7 & 1.6 & 18.5 & 3.4 & 1 \\
\hline
\end{tabular}

cient of variation for $\mathrm{n}_{\mathrm{cr}, \text { rad }}$ is about 0.15 . For $\mathrm{n}_{\mathrm{cr}, \operatorname{tang} \text {, }}$ this value nearly doubles, which indicates a stronger variation of tangential cracks.

The characteristics of fracture patterns for different specimen configurations and the same loading configuration are shown in Table 7. Higher crack densities can be found for specimens with the stiffer interlayers I and
ES. With the increase of tempering, the crack densities increase strongly.

\subsection{Straining in crack center environment}

The evaluation of high speed videos with a DIC software provides the plot of the principal strain as shown 


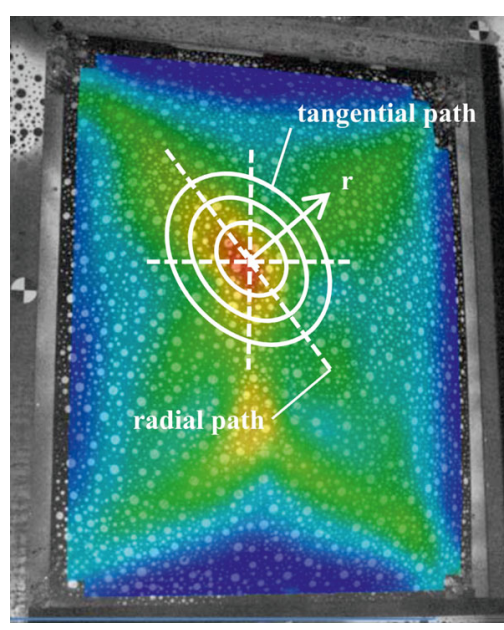

Fig. 10 Plot of principal strain, schematic illustration of tangential and radial paths for further evaluation, courtesy of WTD 52

in Fig. 10. The strain is determined from the measured deformation of the glass surface. In the following, the term strain describes the tensile strain in the Hencky strain measure (true strain). The DIC evaluation facets are chosen in an appropriate size to carry out the measurements even on the fractured glass surface. In consequence, presented strains and strain rates are not related to a specific material component but may be taken as average values for the considered section. In particular, the strain and strain rate of the interlayer will be signif- icantly higher locally between the cracks of the glass. The location of the peak principal strain corresponds to the dominating crack center of the fracture pattern. The strain and the strain rate are evaluated for tangential (solid lines) and radial paths (dashed lines), as they correspond to the directions of principal normal stresses. A tangential path intersects radial cracks in a right angle and owns an elliptical shape. The strain along the paths of three concentric ellipses is determined and related to the short ellipse radius $r$. The evaluation is related to the time from the arrival of the blast wave until total failure or obtainment of maximum encased volume. This period varies between 5 to $35 \mathrm{~ms}$ in different tests. Figures 11, 12 and 13 show the plot of strain and strain rate dependent on $r$ for different testing configurations. The graphs of strain are illustrated with square markers. Those representing the strain rate are highlighted with dot markers.

The strain and the corresponding strain rate in tangential direction from the dominating crack center dependent on $r$ are depicted in Fig. 11 for different configurations of blast-temperature loading. The solid lines represent the specimens tested at ambient temperature. The dashed lines illustrate the tests at elevated temperature. The strain and strain rate are decreasing from the crack center. At the lower testing temperature, the strain along tangential paths is $4-8 \%$. The value increases up to 6-21\% for the elevated temperature level. With regard to the evaluated strain rates,
Fig. 11 Strain and strain rate in tangential direction from dominating crack center versus $r$, test series F-Pr-a and different configurations of blast-temperature loading

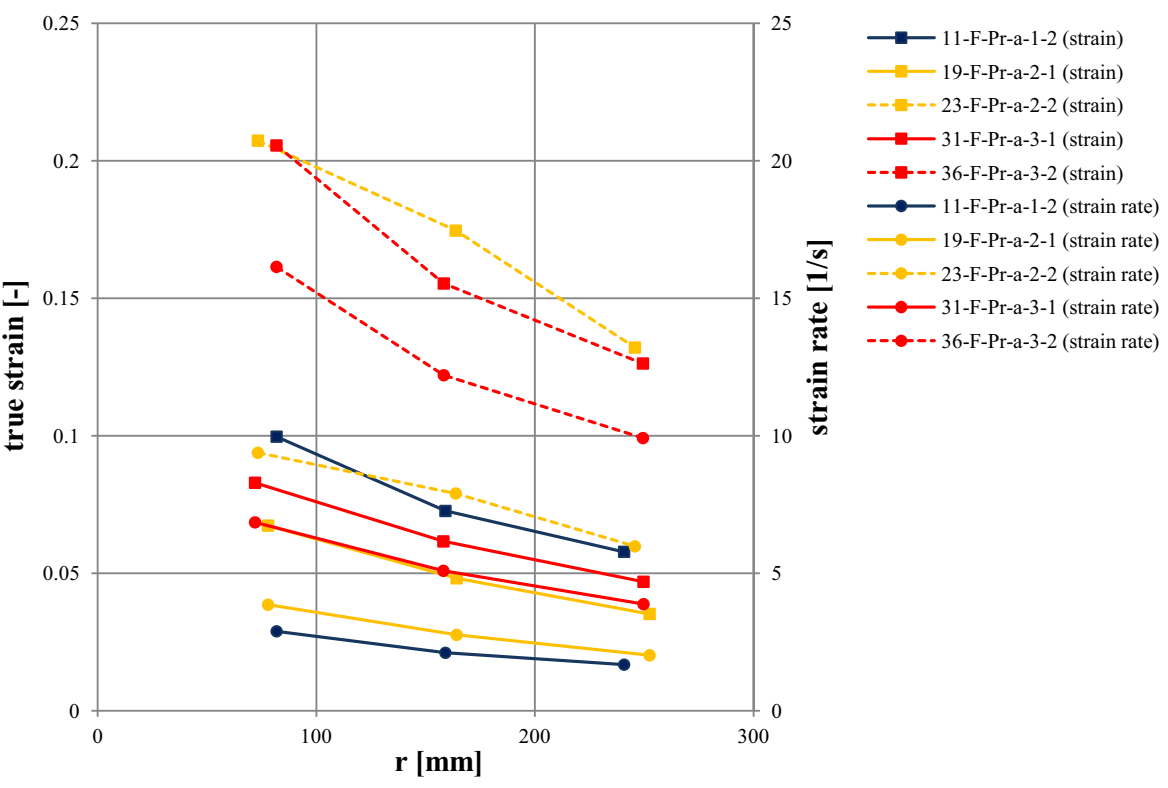


Fig. 12 Strain and strain rate in tangential direction from dominating crack center versus $r$, different interlayers and medium blast load at elevated temperature

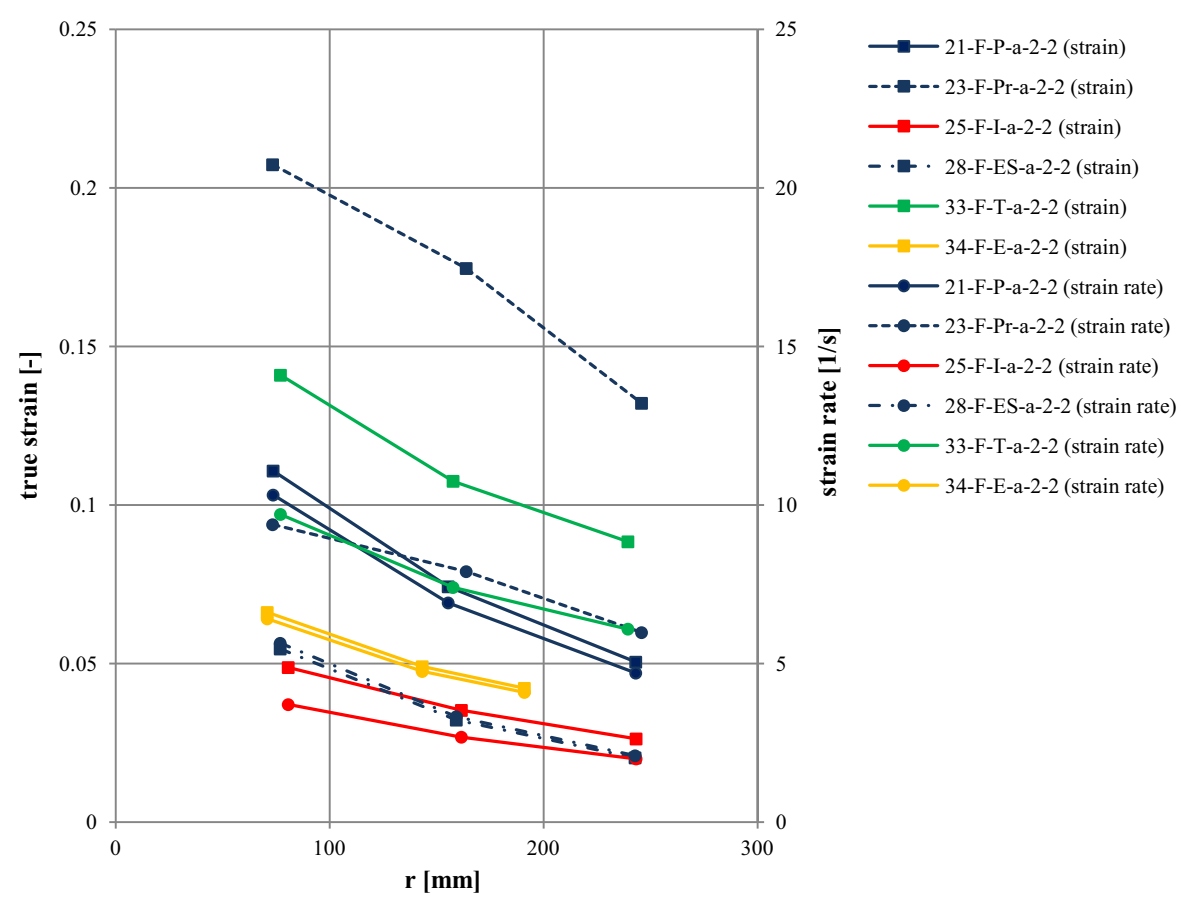

an increase of both the blast load and the temperature load lead to an increased strain rate. The temperatures influences the strain rate more significantly, as the rates double and reach values of up to $17 \mathrm{1} / \mathrm{s}$.

Figure 12 compares strain and strain rate in the same way for different interlayer materials at the same loading configuration. The variation of interlayer materials is illustrated by different colors of the graphs. The specimens with the interlayers P and Pr own nearly the same plot of strain rate; however, the values of strain in comparison to $\mathrm{P}$ are doubled for $\mathrm{Pr}$. The strain rates reach values of up to $101 / \mathrm{s}$. As expected, stiffer interlayers ES and I show the lowest straining.

The strain and strain rate in tangential direction of the specimens with different tempering of the glass component is depicted in Fig. 13. Test 26 is regarded as outlier, since only the glass ply on the protected side of the specimen was fractured after testing. All tests compared in the diagram have been conducted at elevated temperature. The straining and the strain rates increase with stronger tempering of the glass plies. The increase of the blast load leads to a decrease in straining and an increase in the strain rate. The strain rates amount up to $191 / \mathrm{s}$.

Figure 14 depicts the impact of the interlayer temperature $T_{\text {inter,mean }}$ on strain and strain rate from the dominating crack center in radial direction. In any of the test series, a rise of temperature leads to an increase in strain and strain rate. The strongest inclines are determined for test series with specimen configuration F-Pra. The strain rate of F-Pr-a-2-X has tripled from 13 to 30 ${ }^{\circ} \mathrm{C}$. Test series F-I-a-2-X shows less sensitivity within the tested temperature range.

\subsection{Encased volume-time histories}

The dotting, colors and markers of the graphs in the diagrams of this section are similar to the previous section. The encased volume-time histories of the test series with specimens of the interlayer type Pr under different loading configurations are depicted in Fig. 15. For better comparison, the graphs have been shifted in time to start from zero with the initial reaction. Except for the high blast configuration, the evaluation has been stopped by reaching a maximum encased volume. The specimen tested with a low blast load and at low temperature remained elastic and has therefore been excluded in the diagram. An increase of the blast load leads to a faster response of the laminate, attaining an encased volume of around $3-4 \mathrm{E}+07 \mathrm{~mm}^{3}$. Reached volumes exceed those at ambient temperature by far.

Figure 16 compares the encased volume-time histories of specimens with differing interlayer compo- 
Fig. 13 Strain and strain rate in tangential direction from dominating crack center versus $r$, different glass tempering and different configurations of blast-temperature loading

Fig. 14 Strain and strain rate in radial direction intersecting dominating crack center versus

$\mathrm{T}_{\text {inter,mean }}$, different test series
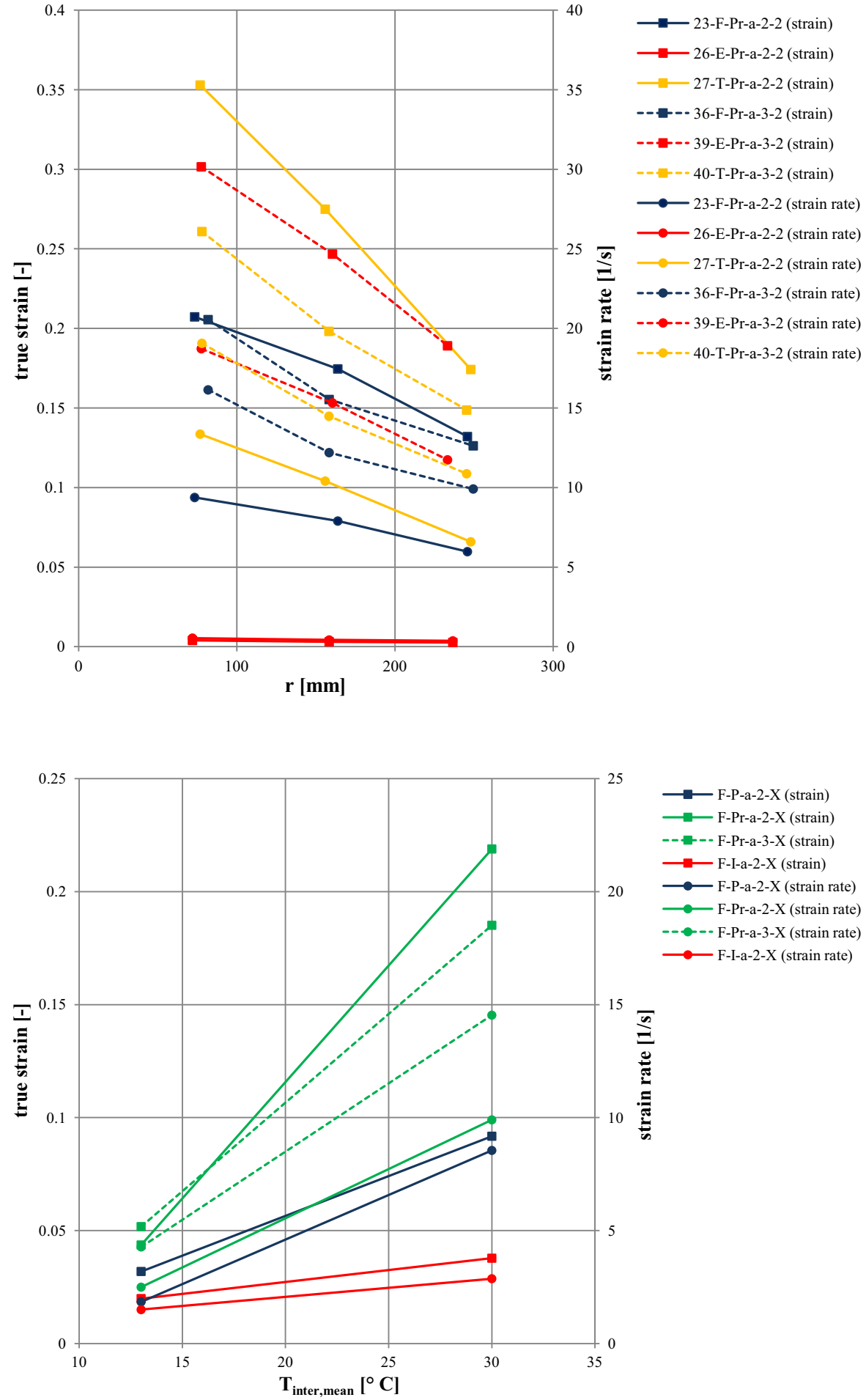

nent at the same loading configuration. Regarding this series, only the specimens of tests 23 and 25 passed the test without an opening in the laminate. This is appar- ent in the gradient decline representing the approach to a maximum deflection before swinging back. Tests with PVB-based interlayers are graphically grouped as 
Fig. 15 Encased volume-time histories for test series F-Pr-a, different configurations of blast-temperature loading

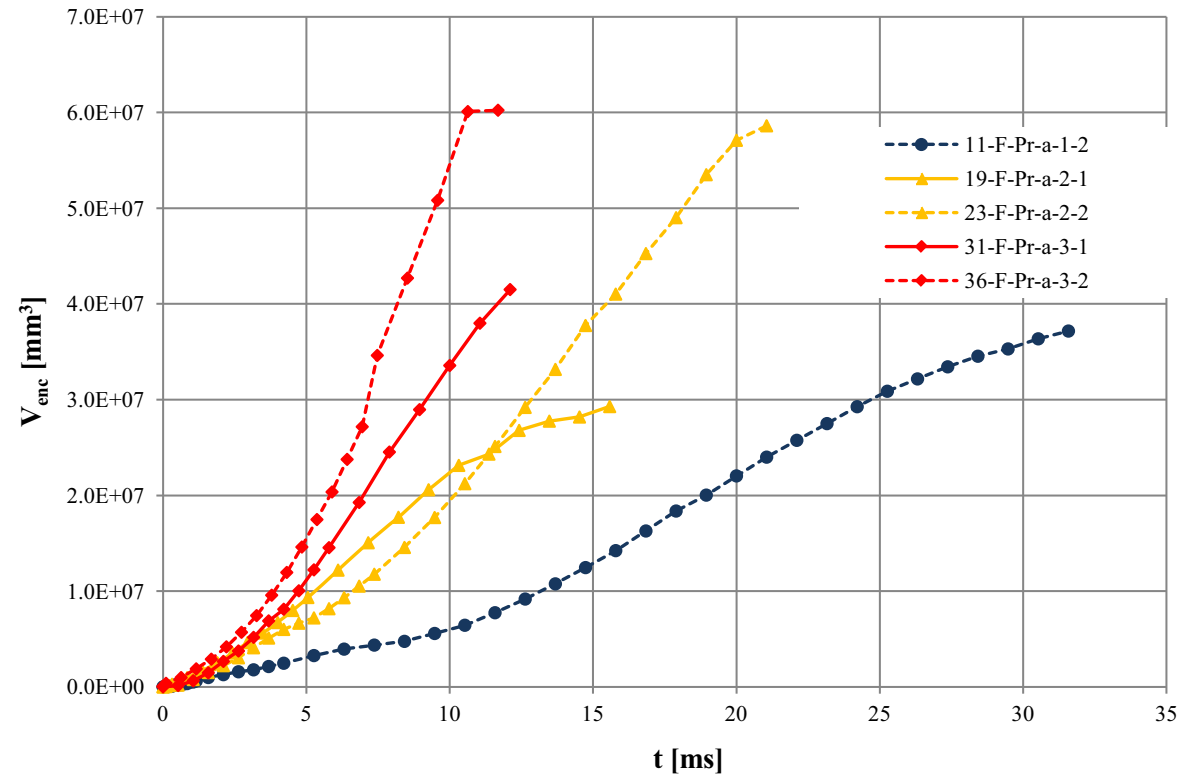

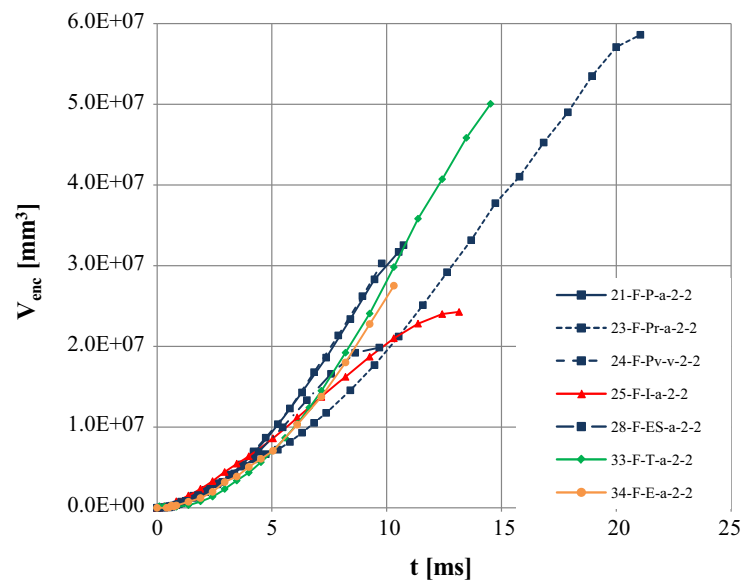

Fig. 16 Encased volume-time histories for specimens with different interlayers, medium blast load at elevated temperature

blue lines with square markers. The curves of specimens with the interlayers $\mathrm{P}$ and $\mathrm{Pv}$ are nearly identical. The specimen of test 23 shows a delayed response and an extensive build-up of encased volume with a maximum of nearly $6.0 \mathrm{E}+07 \mathrm{~mm}^{3}$. Test 23 provides an encased volume of $5.0 \mathrm{E}+07 \mathrm{~mm}^{3}$ before total failure of the laminate. The specimens with the stiffer interlayers I and ES show the lowest encased volumes of the series. However, test 28 has been characterized by a dramatic shear failure of the specimen.

Different tempering of the glass component is compared in Fig. 17. The specimens with fully tempered

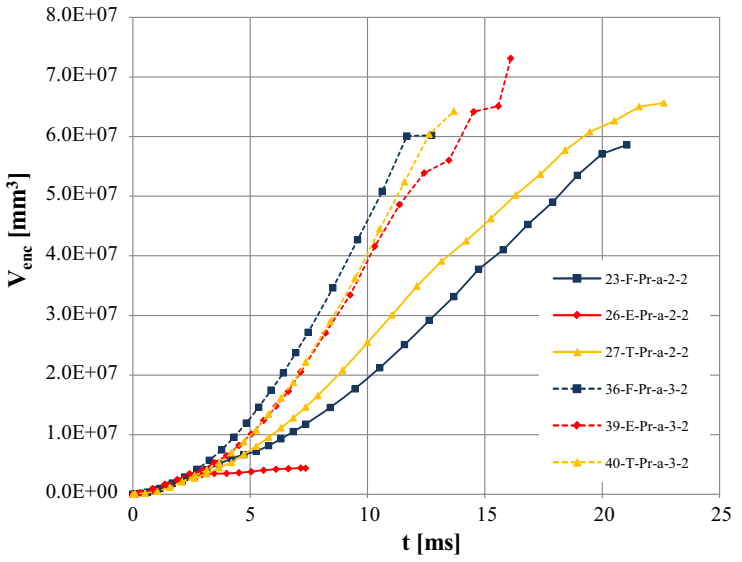

Fig. 17 Encased volume-time histories for specimens of different glass tempering, different configurations of blast-temperature loading

glass plies show the stiffest response. At medium blast load in test 26 , only the protected side of the specimen has been fractured. The influence of the glass tempering becomes less significant at the high blast level. The extensive volume build-up of specimens with the interlayer type Pr is noticed for any test with both glass plies fractured.

For further evaluation according to Equation (2), the encased volume-time histories are differentiated with the central difference quotient method to obtain the time derivative. 


\subsection{Executed internal work in intact state and damaged configurations}

The executed internal work fractions are derived from the evaluation of Eqs. (2) and (3). The evaluation considers the duration from arrival of the blast wave to the moment of interlayer tearing or the moment of reaching a peak deflection, before the swing-back of the laminate takes place. In consequence, the evaluated work portions do not correspond to the final damage state but to a specific damage state on the loading path. The initial occurrence of fracture lines takes place 5 to 15 ms after arrival of the blast wave. In the following the results have to be distinguished regarding tests with total failure of the specimen by tearing of the interlayer (solid bars, $\mathrm{W}_{\mathrm{in}, \mathrm{dmg}}$ fail) and tests with residual load carrying capacity of the specimen (hatched bars, $\mathrm{W}_{\mathrm{in}, \mathrm{dmg}}$ res). The work is given in Kilojoule.

Figure 18 illustrates the fractions of $\mathrm{W}_{\text {in }}$ for test series F-Pr-a. The fractions of the elastic state as well as for damage configurations increase with a higher loading level. This rise is mainly related to $\mathrm{W}_{\mathrm{in}, \mathrm{dmg}}$. $\mathrm{W}_{\text {in,el }}$ increases slightly around $1 \mathrm{~kJ}$, which may be caused by the variation of tensile strength for float glass or by the influence of a loading dependence of the shear modulus. In tests 31 and 36 the execution of work is

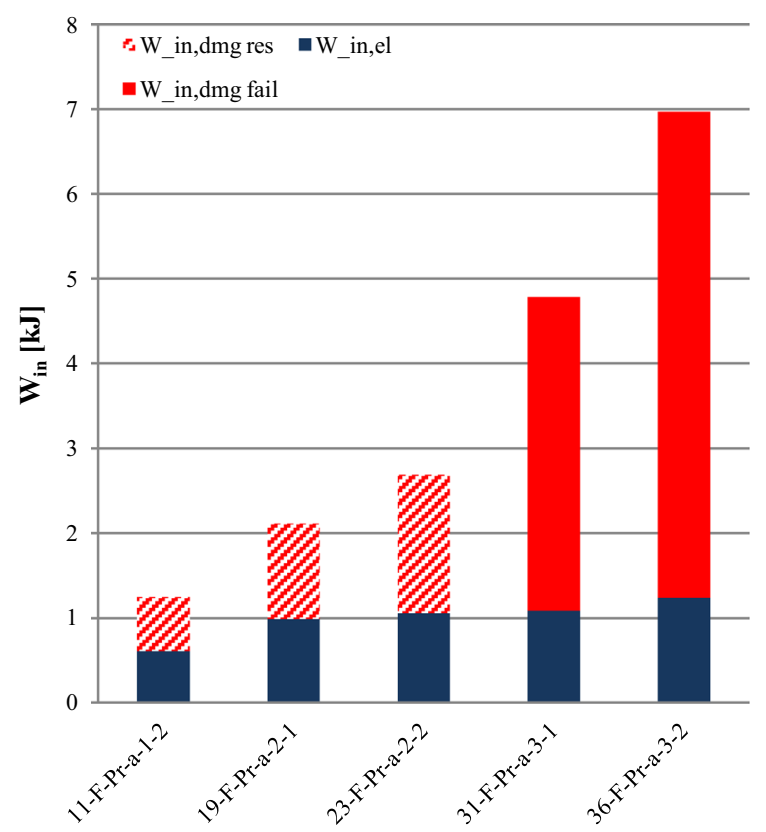

Fig. 18 Fractions of $\mathrm{W}_{\text {in }}$ for test series F-Pr-a, different configurations of blast-temperature loading

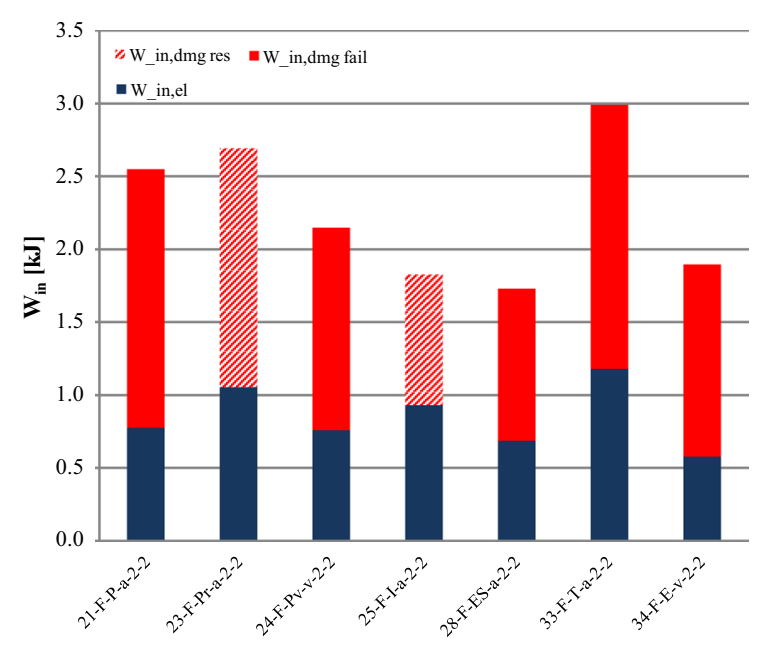

Fig. 19 Fractions of $\mathrm{W}_{\text {in }}$ for specimens with different interlayers, medium blast load at elevated temperature

exploited until tearing of the interlayer. The maximum attainable work executed on the specimen is enhanced at elevated temperature.

The fractions of internal work for specimens with different interlayers at the same loading configuration are compared in Fig. 19. $\mathrm{W}_{\text {in,el }}$ falls within the range of $0.6-1.2 \mathrm{~kJ}$, which may be explained by the scattering of the glass tensile strength. The lowest value of $\mathrm{W}_{\text {in, } \mathrm{dmg}}$ has been obtained in test 25 with $0.9 \mathrm{~kJ}$. The largest amount of work with a value of nearly $3 \mathrm{~kJ}$ has been executed on the specimen of test 33 .

The allocation of work fractions for specimens differing in tempering of the glass component are depicted in Fig. 20. An increase of tempering enhances $W_{\text {in,el }}$ for high blast loading. Work of around $7 \mathrm{~kJ}$ is executed on any of the considered specimens before total failure.

\section{Conclusions}

The experimental investigations show a significant influence of the temperature on the blast resistance of laminated safety glass. The range of realistic temperature loads coincides with different aggregate states of the applied interlayer materials. The elastic response of the composite shows higher displacements at elevated temperature as the shear transfer between the single glass panes is reduced by approaching or partially exceeding the glass transition of the interlayer. A temperature increase leads to a notable intensification of the strains and the strain rates in the crack cen- 


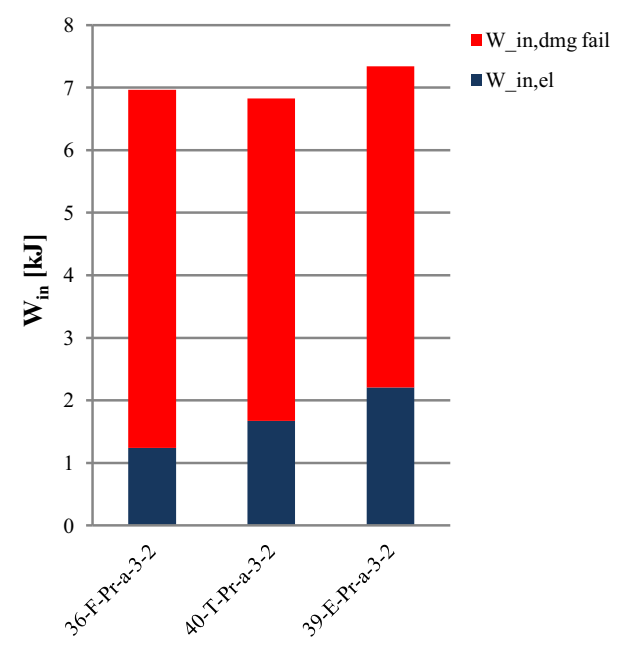

Fig. 20 Fractions of $\mathrm{W}_{\text {in }}$ for specimens of different glass tempering, high blast load at elevated temperature

ter environment. Thereby, average strain rates may be tripled for the same blast loading and reach values of 15 $1 / \mathrm{s}$ in radial directions and $191 / \mathrm{s}$ in tangential directions. Furthermore, the crack densities in radial and tangential direction from a crack center decrease. A lower number of available cracks indicates a decrease of the energy dissipated by glass fracture and reduces the potential of energy dissipation by local delamination. In consequence, a more intensive straining of the interlayer is concentrated on a lower number of crack interfaces. The evaluation of executed internal work fractions according to the presented work balance approach captures the level of exposure and the increase of the elastic resistance by glass tempering. Further investigation is necessary to clarify, if $\mathrm{W}_{\text {in }}$ in the damaged configuration can be taken as measure of residual load carrying capacity. Several parameters have been identified to describe the structural response and in particular the post-fracture behavior of lami- nated safety glass under a combined blast-temperature loading.

For future discussion, Table 8 summarizes the results of conducted shock tube testing by the comparison of five selected specimen performances. In addition to the evaluated parameters, the center displacements are specified in the moment of fracture initiation, $\mathrm{w}_{\mathrm{el}}$, and in the moment of interlayer tearing or reaching the maximum before swing-back, $\mathrm{w}_{\max }$. With regard to the design of a blast resistant glass façade, the question arises, which laminate composition will probably show the best performance. Test 26, 25 and 23 passed the test without an opening. The specimen of test 26 with tempered single glass plies offered the highest crack densities. In combination with a ductile interlayer and a lowered adhesion, the overall response remains stiff with minor ejection of secondary debris. In contrast, the specimen of test 23 with reduced adhesion between the components and float glass shows an extensive deflection, resulting in a major amount of work executed on the specimen. It is assumed, that the potential of energy dissipation in the environment of cracks is exploited. The high hazard of secondary debris observed in the test could be countered by an additional safety film on the protected side of the laminate. Comparing the tests 26 and 23, the laminate with a ductile in-field response will dissipate a higher amount of energy and subsequently transfer less load to the substructure. The choice of a specific composite therefore depends on the approach of capacity balance between the façade elements and the substructure.

The specimen of test 21 with high adhesion between the components showed less ejection of secondary debris than the specimen of test 23 with reduced adhesion but dissipated less energy and failed by tearing of the interlayer from the center. The comparison highlights the role of adhesion and the conflict of secondary

Table 8 Comparison of specimen performance and evaluation parameters

\begin{tabular}{lllll}
\hline Identifier & $\begin{array}{l}\text { Center displacement } \\
\mathrm{W}_{\mathrm{el}} / \mathrm{W}_{\max }[\mathrm{mm}]\end{array}$ & $\begin{array}{l}\mathrm{n}_{\mathrm{cr}, \text { tang }} / \mathrm{n}_{\mathrm{cr}, \text { rad }} \\
{[1 / 10 \mathrm{~cm}]}\end{array}$ & $\begin{array}{l}\text { Max. strain rate, } \\
\text { tangential direction }[1 / \mathrm{s}]\end{array}$ & $\begin{array}{l}\mathrm{W}_{\mathrm{in}, \mathrm{el}} / \mathrm{W}_{\mathrm{in}, \mathrm{dmg}} \\
{[\mathrm{kJ}]}\end{array}$ \\
\hline 26-E-Pr-a-2-2 & $11.0 / 21.2$ & $11.2 / 31.9$ & 0.5 & $0.3 / 0.2$ \\
25-F-I-a-2-2 & $34.0 / 99.7$ & $6.3 / 14.9$ & 3.7 & $0.9 / 0.9$ \\
23-F-Pr-a-2-2 & $40.2 / 222.3$ & $3.9 / 14.7$ & 9.4 & $1.1 / 1.6$ \\
21-F-P-a-2-2 & $26.3 / 125.6$ & $4 / 13.2$ & 10.3 & $0.8 / 1.8$ \\
28-F-ES-a-2-2 & $21.1 / 91.0$ & $6.5 / 15.8$ & 5.6 & $0.7 / 1.0$ \\
\hline
\end{tabular}


debris hazard versus the hazards following an opening in the laminate.

Although the evaluated parameters of tests 25 and 28 are similar, the specimen of test 28 failed by circumferential tearing of the interlayer near the boundary, whereas the specimen of test 25 showed a relatively stiff reaction. This observation in all probability originates from the lower tensile failure strain of the interlayer.

In summary, a dispersed fragmentation of the glass component in combination with a ductile interlayer material is beneficial to the in-field energy dissipation. The fragmentation is notably supported by the glass tempering. A reduced adhesion has a positive impact on the dissipation capacity. The influence of the temperature on the blast performance should be considered in the design process.

Acknowledgments The authors gratefully acknowledge the support of the Bundeswehr Technical Center 52 for conducting the extensive series of full-scale blast testing at Oberjettenberg. Furthermore the support of seele $\mathrm{GmbH}$ for manufacturing and providing the specimens is gratefully acknowledged. On behalf of all authors, the corresponding author states that there is no conflict of interest.

\section{References}

Arruda, E.M., Boyce, C.M.: A three-dimensional constitutive model for the large stretch behavior of rubber elastic materials. J. Mech. Phys. Solids 41(2), 389-412 (1993). doi:10. 1016/0022-5096(93)90013-6

Behr, R.A., Minor, J.E., Norville, H.S.: Structural behavior of architectural laminated glass. J. Struct. Eng. 119(1), 202222 (1993). doi:10.1061/(ASCE)0733-9445(1993)119: $1(202)$

Bjerketvedt, D., Mjaavatten, A.: A hydrogen air explosion in a process plant: a case history. In: Proceedings of the $5^{\text {th }}$ International Conference on Hydrogen Safety, Pisa (2005)

Boyce, C.M., Socrate, S., Llana, P.G.: Constitutive model for the finite deformation stress-strain behavior of poly(ethylene terephthalate) above the glass transition. Polymer 41, 21832201 (2000). doi:10.1016/S0032-3861(99)00406-1

Brismar, B.O., Bergenwald, L.: The terrorist bomb explosion in Bologna, Italy, 1980: an analysis of the effects and injuries sustained. J. Trauma Acute Care Surg. 22(3), 216-220 (1982)

Butchart, C., Overend, M.: Delamination in fractured laminated glass. In: Proceedings of the Third International Conference on Engineered Transparency, pp. 249-257. Düsseldorf (2012)

Cormie, D., Servadio, P., Chang, W., Wholey, W.: Characterizing the pre-fracture response of glazing to blast loads. In: Proceedings of the International Symposium on the Interaction of the Effects of Munitions with Structures, Miramar Beach (2015)
COST Action TU0905, Training School Structural Glass, Darmstadt, 11 March 2013

EN 13541 (German version), Glass in building-security glazing - testing and classification of resistance against explosion pressure. Deutsches Institut für Normung e. V., Beuth, Berlin (2012)

Ensslen, F.: Zum Tragverhalten von Verbund-Sicherheitsglas unter Berücksichtigung der Alterung der PolyvinylbutyralFolie. PhD thesis, Ruhr-Universität Bochum (2005)

Ferretti, D., Rossi, M., Royer-Carfagni, G.: Through cracked tensile delamination test with photoelastic measurements. In: Proceedings of the Challenging Glass Conference 3, pp. 641-652. Delft (2012). doi:10.3233/ 978-1-61499-061-1-641

Franz, J., Schneider, J., Kuntsche, J., Hilcken, J.: Untersuchungen zum Resttragverhalten von Verbundglas: ThroughCracked-Tensile Test. Stahlbau 83(1), 241-252 (2014). doi:10.1002/stab.201490063

Friedlander, G.G.: Note on the Diffraction of Blast Waves by a Wall. UK Home Office Department, RC (A) (1939)

Goebel, H.: Laminated safety glass with EVA-based densely cross-linked interlayer: durability, mechanical and optical oroperties. In: Proceedings of the Glass Performance Days. Tampere (2013)

Henrych, J., Major, R.: The Dynamics of Explosion and Its Use. Elsevier, Amsterdam (1979)

Hooper, J.A.: On the bending of architectural laminated glass. Int. J. Mech. Sci. 14(4), 309-323 (1973). doi:10.1016/ 0020-7403(73)90012-X

Hooper, P.A., Sukhram, R.A.M., Blackman, B.R.K., Dear, J.P.: On the blast resistance of laminated glass. Int. J. Solids Struct. 49, 899-918 (2012). doi:10.1016/j.ijsolstr.2011.12. 008

Iwasaki, R., Sato, C., Latailladeand, J.L., Viot, P.: Experimental study on the interface fracture toughness of PVB (polyvinyl butyral)/glass at high strain rates. Int. J. Crashworthiness 12(3), 293-298 (2007). doi:10.1080/13588260701442249

Juang, L., Lee, L.J., Koelling, K.W.: Rheological analysis of polyvinyl butyral near the glass transition temperature. Polym. Eng. Sci. 41(2), 275-292 (2001). doi:10.1002/pen. 10727

Kinney, G.F., Graham, K.J.: Explosive Shocks in Air. Springer, New York (1985)

Korenev, B., Rabinovič, I.M.: Baudynamik - Konstruktionen unter speziellen Einwirkungen. VEB Verlag für Bauwesen, Berlin (1985)

Kott, A., Vogel, T.: Versuche zum Resttragverhalten von Verbundsicherheitsglas. No. 296, vdf Hochschulverlag AG, Zürich (2006)

Kuntsche, J.: Mechanical behaviour of laminated safety glass under time-dependent and explosion loading. Ph.D. thesis, TU Darmstadt (2015)

Kuraray, Trosifol: Structural PVB film extra strong. http://www. trosifol.de/en/service/downloads/architecture (2015). Accessed 25 Nov 2015

Larcher, M., Solomos, G., Casadei, F., Gebbeken, N.: Experimental and numerical investigations of laminated safety glass subjected to blast loading. Int. J. Impact Eng. 39, 4250 (2012). doi:10.1016/j.ijimpeng.2011.09.006

Martinsen, W. E., Marx, J. D.: An improved model for the prediction of radiant heat from fireballs. In: Proceedings of the 
International Conference and Workshop on Modeling the Consequences of Accidental Releases of Hazardous Materials, San Francisco (1999)

Morison, C.: The resistance of laminated glass to blast loading and the coefficients for single degree of freedom analysis of laminated glass. Ph.D. thesis, Cranfield University (2010)

Mueller, K., Yokoyama, T., Marjanishvili, S., Quiel, S.: Structural response to blast and subsequent fire from a petrochemical train derailment. In: Proceedings of the 1st International Conference on Structural Safety under Fire \& Blast, pp. 319-328. Glasgow (2015)

Muralidhar, S., Jagota, S., Bennison, S.J., Saigal, S.: Mechanical behaviour in tension of cracked glass bridged by an elastomeric ligament. Acta Mater. 48, 4577-4588 (2000). doi:10.1016/S1359-6454(00)00244-5

Norville, H.S., Harvill, N., Conrath, E.J., Shariat, S., Mallonnee, S.: Glass-related injuries in Oklahoma City bombing. J. Perform. Constr. Facil. 13(2), 50-56 (1999). doi:10.1061/ (ASCE)0887-3828(1999)13:2(50)

Norville, H.S., King, K.W., Swofford, J.L.: Behaviour and strength of laminated glass. J. Eng. Mech. 124(1), 46-53 (1998). doi:10.1061/(ASCE)0733-9399(1998)124:1(46)

Palermo, D., Liu, C.: Blast resistant glazing designs of urethane elastomers. In: Proceedings of the 3rd International Conference on Protective Structures, pp. 401-409. Newcastle (2015)

Sackmann, V.: Untersuchungen zur Dauerhaftigkeit des Schubverbunds in Verbundsicherheitsglas mit unterschiedlichen Folien aus Polyvinylbutyral. Ph.D. thesis, TU München (2008)

Salas, M.D., Iollo, A.: Entropy jump across an inviscid shock wave. Theor. Comput. Fluid Dyn. 8, 365-375 (1996). doi:10.1007/BF00456376

Schuler, C.: Einfluss des Materialverhaltens von Polyvinylbutyral auf das Tragverhalten von Verbund-Sicherheitsglas in Abhängigkeit von Temperatur und Belastung. Ph.D. thesis, TU München (2003)

Schuster, M.: Untersuchung des zeitabhängigen Materialverhaltens von VSG-Zwischenschichten. Master thesis, TU Darmstadt (2014)
Schwarzl, F.R.: Polymermechanik - Struktur und mechanisches Verhalten von Polymeren. Springer, Berlin (1990)

SentryGlas SG5000, ionoplast interlayer: technical datasheet. http://glasslaminatingsolutions.kuraray.com/fileadmin/user _upload/technical_information/downloads/sentryglas/150 129_Kuraray_TM_Datenblatt_SG.pdf (2015). Accessed 25 Nov 2015

Seshadri, M., Bennison, S.J., Jagota, A., Saigal, S.: Mechanical response of cracked laminated plates. Acta Mater. 50, 44774490 (2002). doi:10.1016/S1359-6454(02)00255-0

Sha, Y., Hui, C.Y., Kramer, E.J., Garrett, P.D., Knapczy, J.W.: Analysis of adhesion and interface debonding in laminated safety glass. J. Adhes. Sci. Technol. 11(1), 49-63 (1997). doi:10.1163/156856197X01010

Sobek, W., Kutterer, M., Messmer, R.: Untersuchungen zum Schubverbund bei Verbundsicherheitsglas - Ermittlung des zeit- und temperaturabhängigen Schubmoduls von PVB. Bauingenieur 75(1), 41-46 (2000)

Vallabhan, C.V.G., Das, Y.C., Magdi, M., Asik, M., Bailey, J.R.: Analysis of laminated glass units. J. Struct. Eng. 119(5), 1572-1585 (1993). doi:10.1061/ (ASCE)0733-9445(1993)119:5(1572)

Wei, J., Shetty, M.S., Dharani, L.R.: Stress characteristics of a laminated architectural glazing subjected to blast loading. Comput. Struct. 84, 699-707 (2006). doi:10.1016/j. compstruc.2005.11.007

Wellershoff, F., Teich, M., Nehring, G., Gebbeken, N.: Konstruktion und Berechnung von explosionshemmenden Seilnetzfassaden. Stahlbau 81(1), 13-25 (2012). doi:10.1002/stab. 201201501

Williams, M.L., Landel, R.F., Ferry, J.D.: The temperature dependence of relaxation mechanisms in amorphous polymers and other glass-forming liquids. J. Am. Chem. Soc. 77(14), 3701-3707 (1955). doi:10.1021/ja01619a008

Zhang, X.: Development of design and analysis methods for window structures against blast and impact loads. Ph.D. thesis, University of Western Australia, Crawley (2014) 